\title{
Antigen-activated dendritic cells ameliorate influenza $A$ infections
}

\author{
Kobporn Boonnak, ${ }^{1}$ Leatrice Vogel, ${ }^{1}$ Marlene Orandle, ${ }^{2}$ Daniel Zimmerman, ${ }^{3}$ \\ Eyal Talor, ${ }^{3}$ and Kanta Subbarao ${ }^{1}$
}

${ }^{1}$ Laboratory of Infectious Diseases and ${ }^{2}$ Comparative Medicine Branch, National Institute of Allergy and Infectious Diseases (NIAID), $\mathrm{NIH}$, Bethesda, Maryland, USA. ${ }^{3} \mathrm{CEL}-\mathrm{SCI}$ Corp., Vienna, Virginia, USA.

\begin{abstract}
Influenza A viruses cause significant morbidity and mortality worldwide. There is a need for alternative or adjunct therapies, as resistance to currently used antiviral drugs is emerging rapidly. We tested ligand epitope antigen presentation system (LEAPS) technology as a new immune-based treatment for influenza virus infection in a mouse model. Influenza-J-LEAPS peptides were synthesized by conjugating the binding ligand derived from the $\beta_{2}$-microglobulin chain of the human MHC class I molecule (J-LEAPS) with 15 to 30 amino acid-long peptides derived from influenza virus NP, $M$, or HA proteins. DCs were stimulated with influenzaJ-LEAPS peptides (influenza-J-LEAPS) and injected intravenously into infected mice. Antigen-specific LEAPSstimulated DCs were effective in reducing influenza virus replication in the lungs and enhancing survival of infected animals. Additionally, they augmented influenza-specific $T$ cell responses in the lungs and reduced the severity of disease by limiting excessive cytokine responses, which are known to contribute to morbidity and mortality following influenza virus infection. Our data demonstrate that influenza-J-LEAPS-pulsed DCs reduce virus replication in the lungs, enhance survival, and modulate the protective immune responses that eliminate the virus while preventing excessive cytokines that could injure the host. This approach shows promise as an adjunct to antiviral treatment of influenza virus infections.
\end{abstract}

\section{Introduction}

Therapeutic strategies designed to combat influenza rely primarily on antiviral drugs (1). Although these drugs are effective in uncomplicated influenza infections (2), their efficacy is limited in severe cases of influenza (3), including highly pathogenic avian influenza A H5N1 virus infections, which are known to be associated with a high case-fatality rate (4). The high case-fatality rate is complicated by the rapid emergence of antiviral resistance (5), which can render conventional antiviral therapy ineffective (6-8). Thus, there is interest in novel therapeutic approaches for the effective treatment of severe influenza virus infections. We explored the use of ligand epitope antigen presentation system (LEAPS) technology in which short peptides derived from influenza proteins are covalently attached to an immune-binding ligand and are used to activate DCs that are then injected into the host. It has been shown previously that viral peptides covalently attached to carrier peptides derived from the $\beta_{2}$-immunoglobulin chain of the MHC class I molecule (J-LEAPS) (9-13), which is a highly conserved peptide sequence across many different mammalian species (14), can convert the short peptides into immunogens and elicit protection from infectious challenge (15-17).

We covalently conjugated peptides derived from influenza virus nucleoprotein (NP), ion channel protein (M2e), and HA protein to J-LEAPS (referred to as influenza-J-LEAPS), used them to pulse and activate murine BM-derived DCs (BMDCs), and delivered the pulsed DCs i.v. to influenza virus-infected mice. We demonstrate that this treatment is effective in reducing influenza virus replication in the lungs and enhancing survival of infected animals. We found that influenza-J-LEAPS-activated DCs augment influenza-specific T cell responses at the site of infection and limit excessive cytokine

Conflict of interest: The authors have declared that no conflict of interest exists. Citation for this article: J Clin Invest. 2013;123(7):2850-2861. doi:10.1172/JCI67550. responses (cytokine storm), which is a key contributor to the morbidity and mortality associated with influenza virus infection (18-20). Treatment with J-LEAPS conjugates triggers Th1 responses, as shown previously in prophylaxis for herpes simplex virus (HSV) $(15-17,21)$. Other studies with J-LEAPS conjugates support their therapeutic use for autoimmune conditions $(22,23)$. In this study, we have explored whether, when, and how treatment of influenza with LEAPS-pulsed DCs can ameliorate disease and death.

\section{Results}

Influenza-J-LEAPS promotes DC maturation. Maturation of DCs is critical for antigen presentation and stimulation of $T$ cell responses. We first determined whether influenza-J-LEAPS (Supplemental Table 1; supplemental material available online with this article; doi:10.1172/JCI67550DS1) would trigger maturation of DCs. BM progenitor cells were cultured in the presence of murine GM-CSF. DC morphology was observed in almost all cells on day 8 , with $90 \%-95 \%$ viability; DC phenotypes were confirmed by monitoring cell-surface markers. We observed high levels of CD11c, CD86, and MHC-II, with undetectable levels of CD3, CD19, and F4/80 on the cell surface (Supplemental Figure 1), suggesting that a majority of cells were differentiated into DCs. DC maturation markers (CD80 hi, CD86 ${ }^{\text {hi }}$, MHC-II ${ }^{\text {hi }}$ ) were observed 24 hours after culturing DCs in the presence of influenza-J-LEAPS (Supplemental Figure 2). We observed that upregulation of MHC-II and CD86 on DCs with peptide-conjugated LEAPS resulted in a bimodal population. The DCs that expressed high levels of CD86 also expressed high levels of MHC-II (Supplemental Figure 3A). We tested the ability of individual influenza-J-LEAPS peptides to induce maturation of DCs and found that stimulation with M2e, NP, and combined influenza peptides conjugated to LEAPS resulted in better DC maturation than HA1- and HA2-conjugated LEAPS peptides (HA1-LEAPS 
A
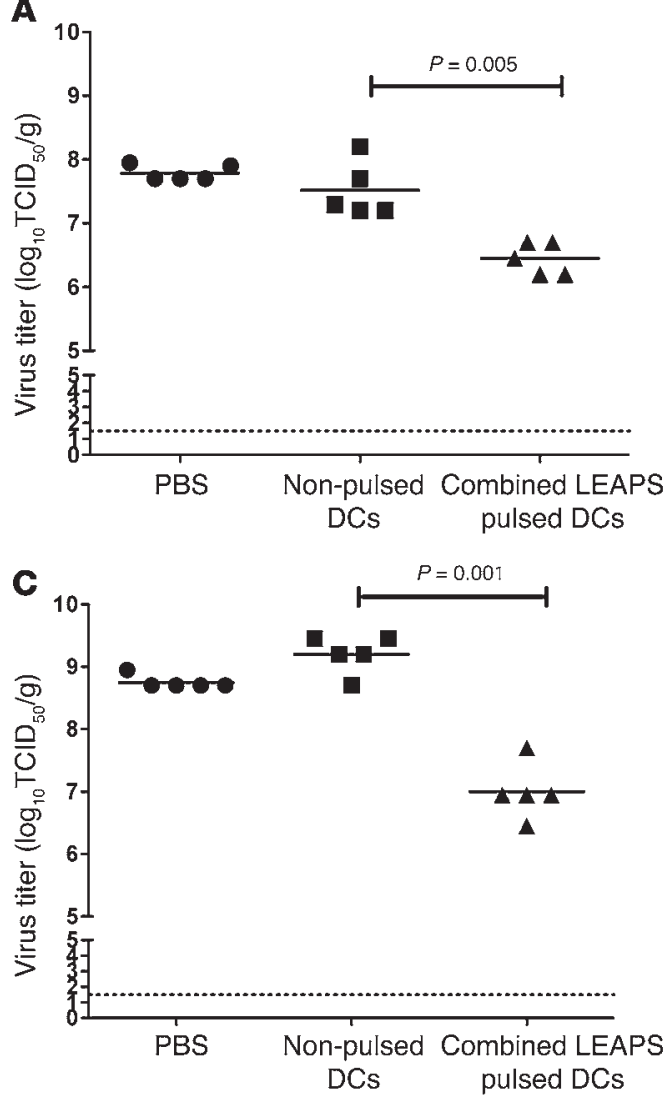

B

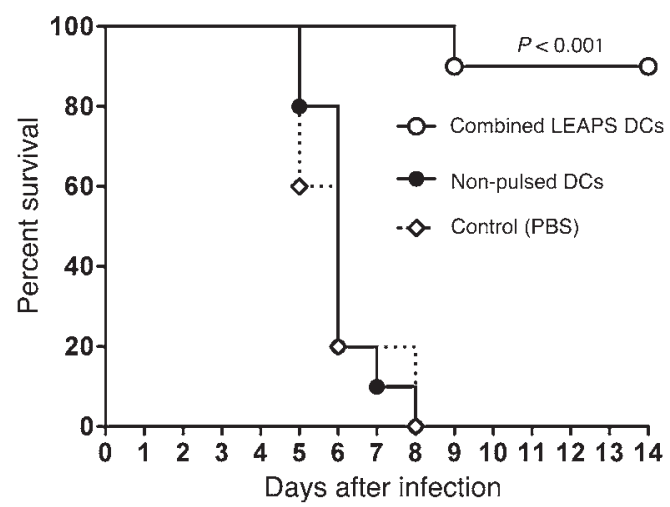

D

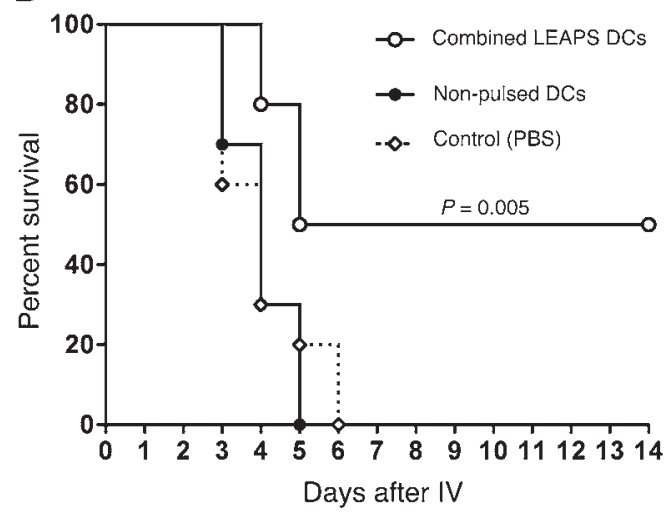

Figure 1

Combined influenza-J-LEAPS-loaded DCs reduce morbidity and mortality in influenza virus-infected mice. BALB/c mice were infected i.n. with $10 \mathrm{LD}_{50}(\mathbf{A}$ and $\mathbf{B})$ or $1000 \mathrm{LD}_{50}(\mathbf{C}$ and $\mathbf{D})$ of PR8. Twenty-four hours after infection, the mice were given an i.v. injection of PBS, nonpulsed DCs, or a combination of NP-, HA- and M2e-conjugated LEAPS-pulsed DCs. Virus titers were measured in the lungs harvested 4 days after infection. $P=0.005$, Student's $t$ test for low-dose infection $(\mathbf{A}) ; P=0.001$, Student's $t$ test for high-dose infection (C). The mean titer from each group is represented as a solid bar. The dashed line represents the lower limit of detection. Percentage survival following infection was monitored for 14 days; $P \leq 0.001$ for low-dose infection (B); $P=0.005$ for high-dose infection (D), log-rank (Mantel-Cox) test.

and HA2-LEAPS, respectively) (Supplemental Figure 3B). Therefore, we used combined peptides or NP-conjugated LEAPS (NP-LEAPS) for further experiments.

Influenza-J-LEAPS-pulsed DCs rescue mice from lethal influenza infection. To determine the effect of LEAPS-pulsed DCs on influenza infection, we infected mice i.n. with a lethal dose $\left(10^{3} \mathrm{TCID}_{50}\right.$; $10 \mathrm{LD}_{50}$ ) of the mouse-adapted influenza virus A/Puerto Rico/8/34 (H1N1) (PR8). Treatment of 3 groups of mice was initiated 24 hours after infection by administering $200 \mu \mathrm{l}$ of $10^{7}$ nonpulsed DCs, combined LEAPS (HA, NP, and M2e) pulsed DCs, or $200 \mu \mathrm{l}$ of sterile PBS i.v. Three days later, the lungs of mice from the 3 groups were harvested for virus titration. Lower virus titers were observed in the lungs of mice that received combined-LEAPSpulsed DCs than in mice that received nonpulsed DCs or PBS $(P=0.005$; Figure $1 \mathrm{~A})$. Improved survival was also observed in mice that received combined-LEAPS treatment; $90 \%$ of the animals in this group survived for 14 days $(P<0.001$; Figure 1B). We also determined whether LEAPS-stimulated DCs would protect mice from challenge with a higher dose of virus. We infected mice with $10^{5} \mathrm{TCID}_{50}\left(1000 \mathrm{LD}_{50}\right)$ of virus and administered combinedLEAPS-pulsed DCs 24 hours after infection. Again, lungs were harvested 4 days after initial infection (corresponding to 3 days after DC injection), and lower virus titers were seen in the lungs of combined-LEAPS-treated mice than in mice that received nonpulsed DCs $(P=0.001$; Figure $1 C)$. With this higher dose of virus, $50 \%$ of the mice that received combined-LEAPS treatment survived $(P=0.005$; Figure 1D). These data indicate that LEAPS treatment significantly reduced the titer of virus in the lungs and mortality in influenza virus-infected mice.

Influenza-J-LEAPS-pulsed DCs home to the lungs of influenza-infected mice. We designed an experiment to determine the localization of the injected influenza-J-LEAPS-pulsed DCs in uninfected and infected mice. Mice were inoculated i.n. with $10 \mathrm{LD}_{50}\left(10^{3} \mathrm{TCID}_{50}\right)$ of PR8 virus or diluent (Leibovitz-15, L-15) 24 hours before administering $10^{7}$ cells of CFSE-labeled nonpulsed or combined-LEAPSpulsed DCs i.v. The numbers of CFSE-positive cells were determined in the lungs 8,24 , and 48 hours later. We did not detect significant numbers of CFSE-labeled nonpulsed, combined-LEAPS-pulsed DCs or DCs pulsed with an irrelevant peptide (JH-LEAPS) in the lungs of uninfected animals (Figure 2A). Interestingly, CFSElabeled combined-LEAPS-pulsed DCs, but not nonpulsed DCs or JH-LEAPS-pulsed DCs, began to accumulate in the lungs of infected animals by 8 hours and remained detectable in the lungs 24 hours following injection (Figure 2B). We did not observe signifi- 
A
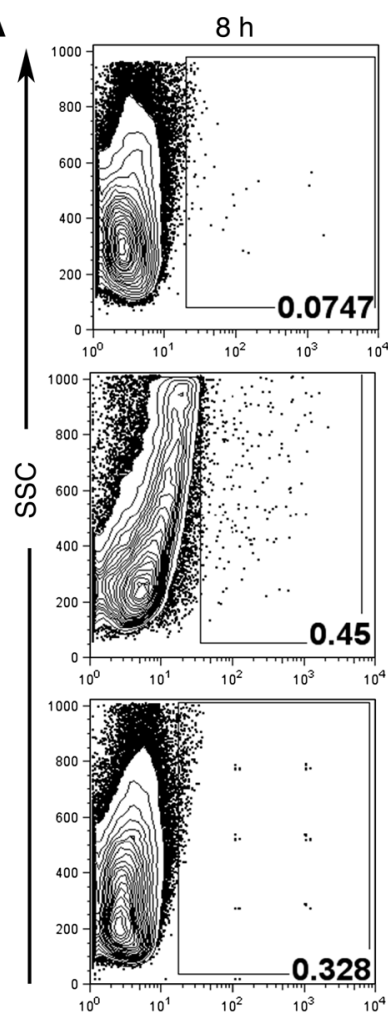

B
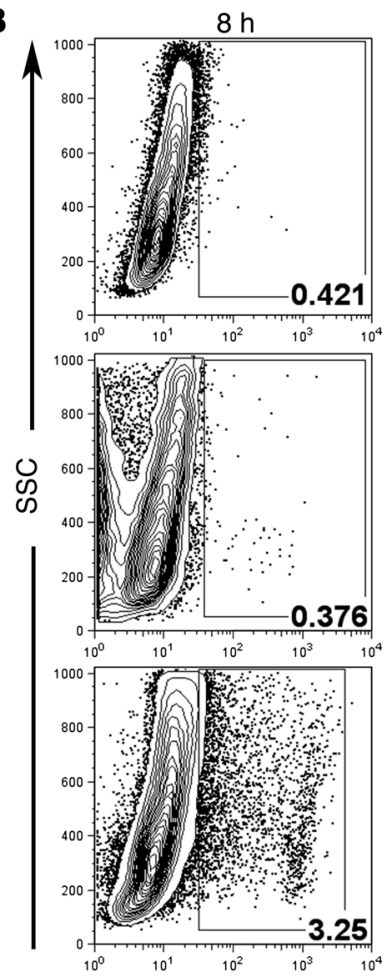
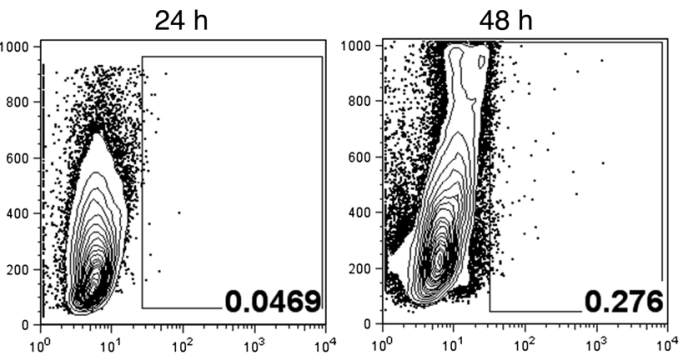

Non-pulsed DCs
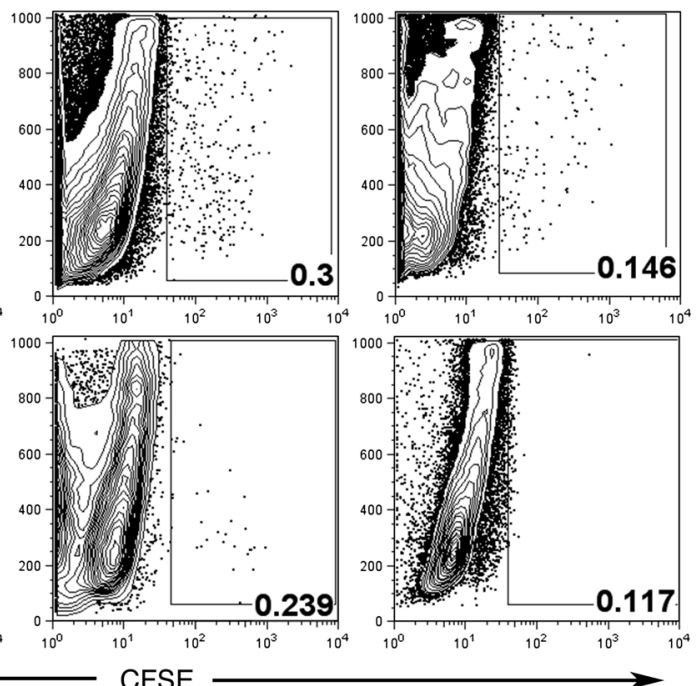

JH LEAPS-pulsed DCs

Combined-LEAPS-pulsed DCs

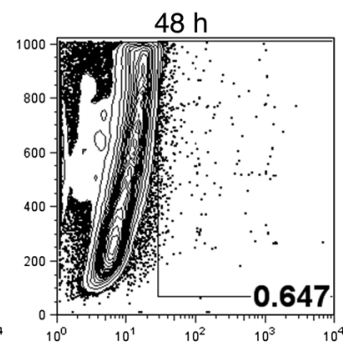

Non-pulsed DCs
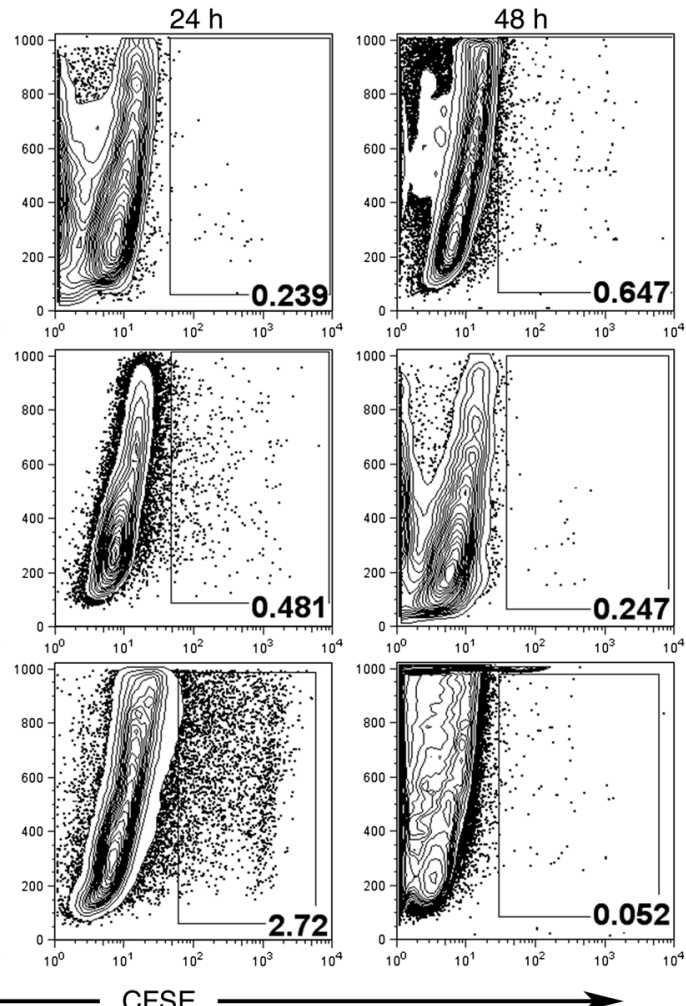

JH LEAPS-pulsed DCs

Combined-LEAPS-pulsed DCs

Figure 2

LEAPS-loaded DCs target the site of infection. $10^{7}$ CFSE-labeled nonpulsed DCs, JH-LEAPS-pulsed DCs (irrelevant LEAPS control), or combined-LEAPS-pulsed DCs were injected i.v. into naive mice $(\mathbf{A})$ or influenza virus-infected mice $\left(10 \mathrm{LD}_{50}\right.$, PR8 virus, i.n.) (B). The accumulation of CFSE-positive cells was measured in the lungs of mice 8,24 , and 48 hours after administration of the LEAPS-stimulated DCs. The data are representative FACS plots from each group ( $n=5 \mathrm{mice} / \mathrm{group}$ ). 
A
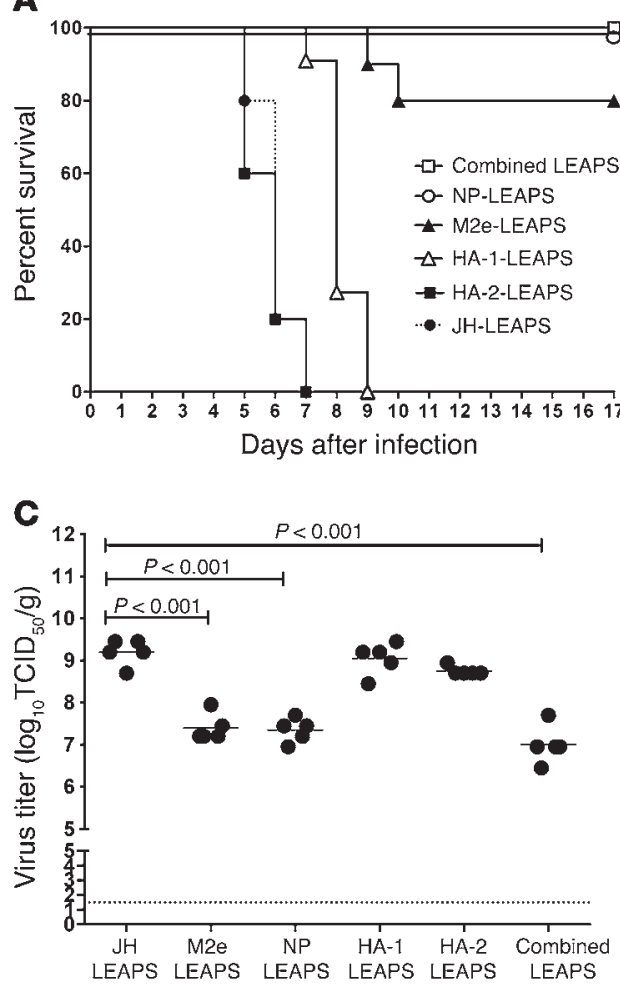

$\longrightarrow$ Peptides used to pulse DCs $\longrightarrow$

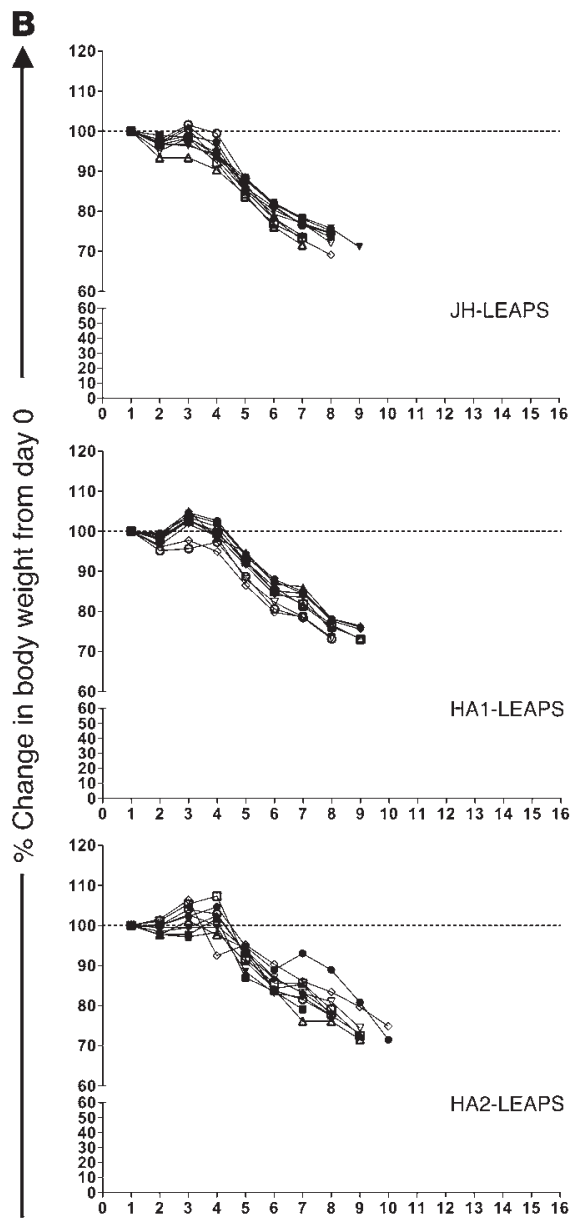

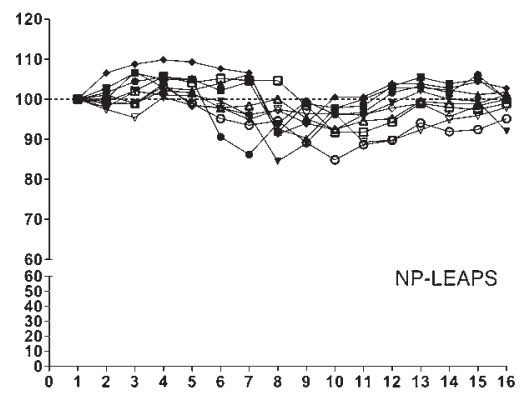
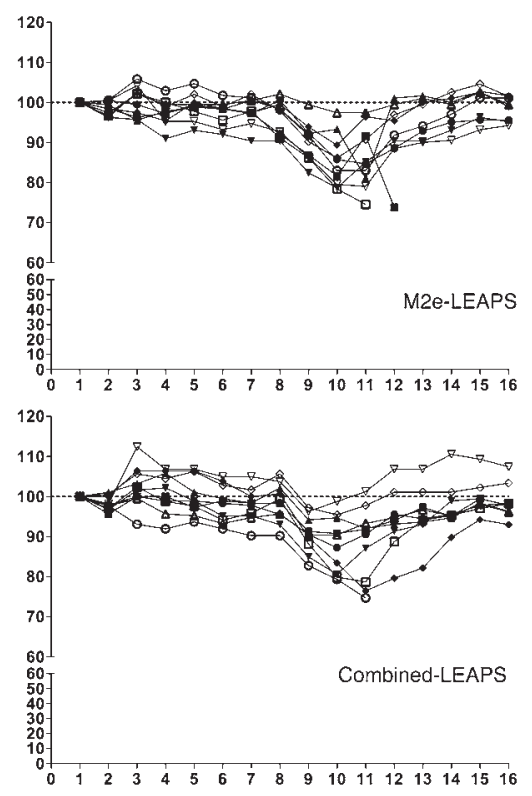

Days after infection

\section{Figure 3}

M2e and NP peptides are critical determinants of efficacy of LEAPS treatment. BALB/c mice were infected i.n. with 10 LD $_{50}$ of PR8. Twenty-four hours after infection, mice received NP, HA1, HA2, or M2e-conjugated LEAPS pulsed DCs. LEAPS conjugated with a combination of these peptides was used as a positive control and JH-LEAPS was used as negative control. Percentage survival (A), percentage change in body weight (B), and virus titer in lungs harvested 4 days after infection are presented $(\mathbf{C})$. In $\mathbf{C}$, the mean titer from each group is represented as a solid bar and the dashed line represents the lower limit of detection.

cant numbers of CFSE-labeled DCs in the lymph nodes or spleen of uninfected or infected animals (data not shown), and similar levels of CFSE-labeled DCs were observed in whole blood obtained from naive and PR8-infected mice that received nonpulsed DCs or JH-LEAPS-pulsed DCs (Supplemental Figure 4). Interestingly, the levels of CFSE-labeled DCs in the whole blood of naive mice were higher than in PR8-infected mice that received combined LEAPSpulsed DCs, presumably because $\mathrm{CFSE}^{+}$DCs homed to the lungs in infected animals (Supplemental Figure 4). These findings suggest that LEAPS stimulation enhances the homing of influenza-peptidebearing DCs to the lungs, which is the site of infection. We further asked whether LEAPS-stimulated DCs enhanced maturation of endogenous DCs. The expression of CD86 and MHC-II were determined on the cell surface of endogenous DCs (CFSE-CD11 $\mathrm{c}^{+}$) in the lungs of naive and infected mice 24 hours following i.v. injection of CFSE-labeled combined-LEAPS-pulsed DCs. The endogenous DCs $\left(\mathrm{CFSE}^{-} \mathrm{CD} 11 \mathrm{c}^{+}\right)$of infected mice that received CFSE-labeled combined LEAPS-pulsed DCs expressed high levels of CD86 and MHC-II, but those of naive mice did not (Supplemental Figure 5).
Therapy with NP-LEAPS, M2e-LEAPS, and combined-LEAPS-pulsed $D C$ reduces viral replication in the lungs and improves survival of influenza virus-infected mice. We next investigated which of the influenza peptide(s) were critical for the observed efficacy of the combinedLEAPS DC treatment. We stimulated DCs with individual influenza peptides (HA1, HA2, NP, or M2e) conjugated to LEAPS (Supplemental Table 1). We infected 6 groups of mice with a low dose $\left(10^{3}\right.$ $\left.\mathrm{TCID}_{50}, 10 \mathrm{LD}_{50}\right)$ of PR8 and administered $10^{7} \mathrm{DCs}$ pulsed with NPLEAPS, M2e-LEAPS, HA1-LEAPS, HA2-LEAPS, combined-LEAPS or JH-LEAPS i.v. 24 hours later. NP-LEAPS-, M2e-LEAPS-, and combined-LEAPS-pulsed DCs showed a similar effect on survival (Figure 3A), weight loss (Figure 3B), and virus titers in the lungs of infected animals 4 days after infection (Figure 3C). These data indicate that either NP and/or M2e is critical for the efficacy of LEAPS treatment. DCs pulsed with HA1 or HA2 peptides provided no benefit in restriction of virus replication or morbidity and mortality (Figure 3). Because the influenza peptides selected for this study are conserved between PR8 and the 2009 pandemic influenza virus, A/California/07/2009 (H1N1) (pH1N1) (Supplemental Table 1), 

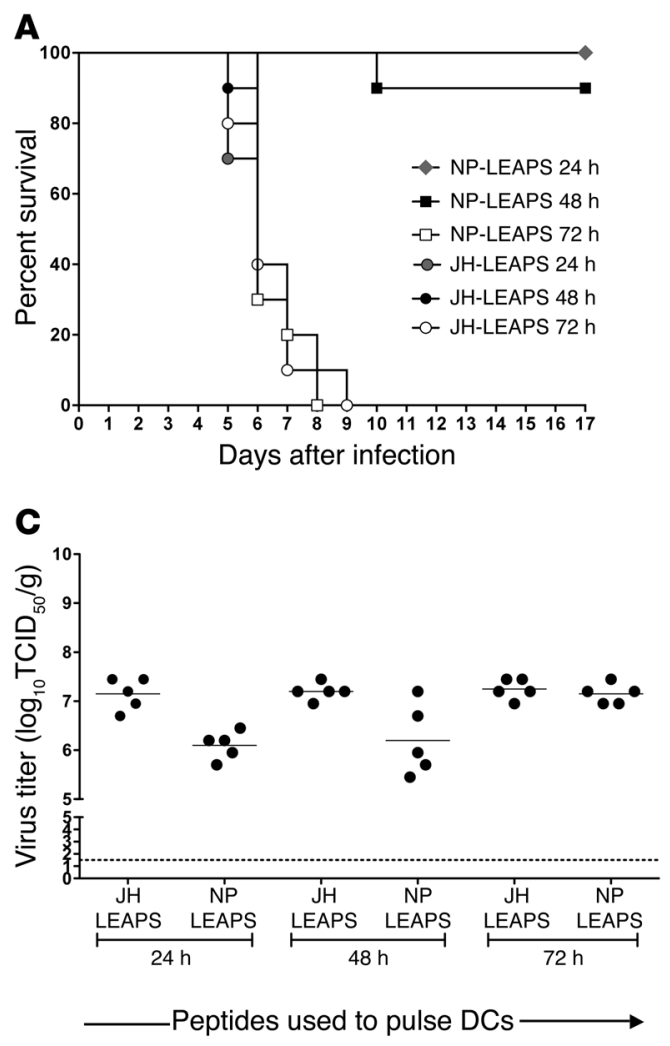

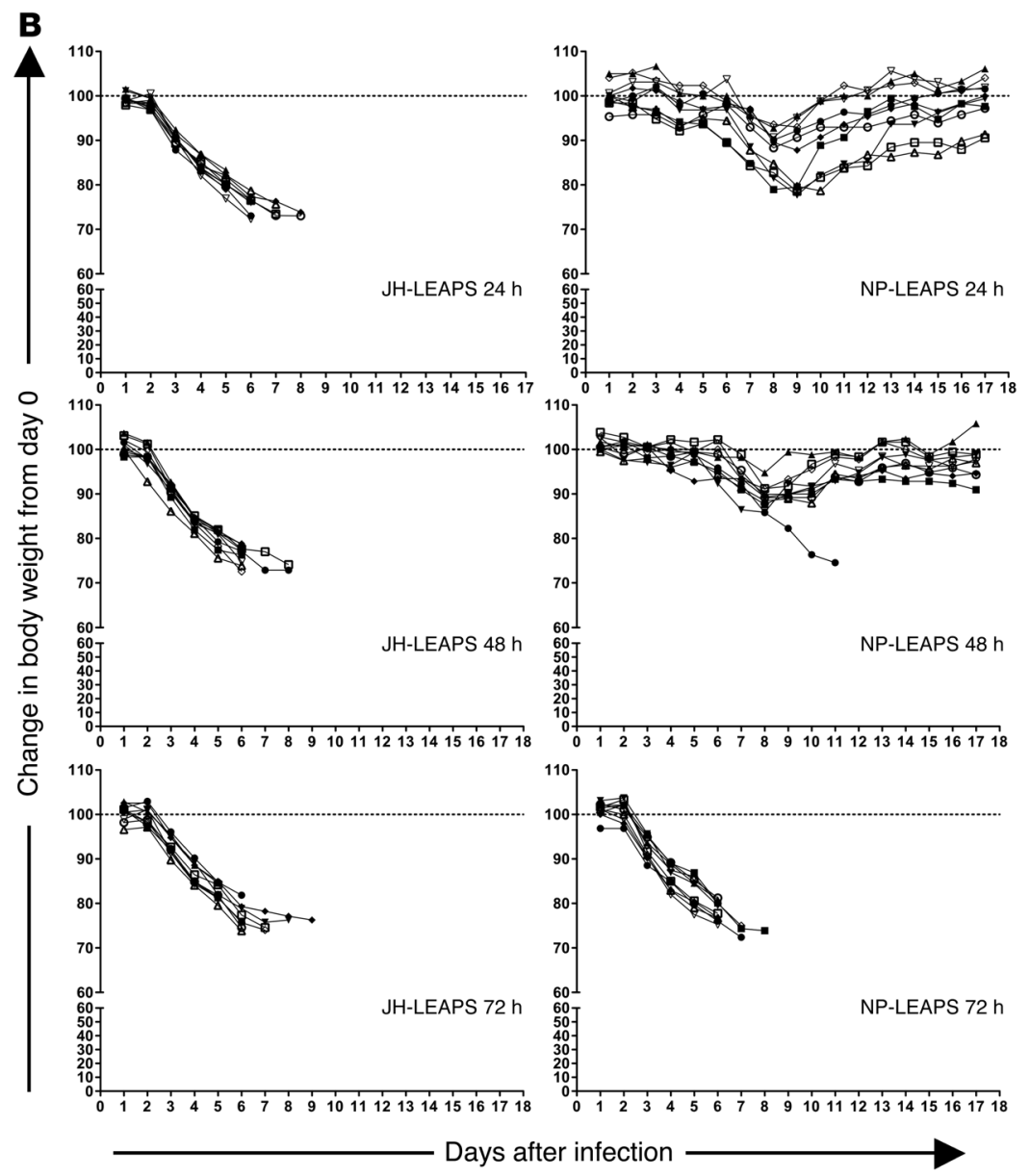

\section{Figure 4}

LEAPS treatment is effective when administered at 24 or 48 hours after infection. Mice infected with 10 LD 50 of PR8 received an i.v. injection of NP-LEAPS - or JH-LEAPS-pulsed DCs injected at 24, 48, or 72 hours after infection. Percentage survival (A), percentage change in body weight (B), and virus titer in the lungs 4 days after infection are presented (C). In C, the mean titer from each group is represented as a solid bar, and the dashed line represents the low limit of detection.

we also investigated whether LEAPS treatment could rescue mice from lethal $\mathrm{pH} 1 \mathrm{~N} 1$ infection. We observed significantly reduced virus titers in the lungs $(P=0.001)$ associated with increased survival and reduced weight loss in $\mathrm{pH} 1 \mathrm{~N} 1$-infected mice that received combined-LEAPS treatment (Supplemental Figure 6).

Influenza-J-LEAPS treatment is effective when administered at 24 or 48 hours after infection. We next determined the optimal time for influenza-J-LEAPS treatment. We infected mice with a low dose $\left(10^{3} \mathrm{TCID}_{50}, 10 \mathrm{LD}_{50}\right)$ of PR8, which results in $100 \%$ mortality between days 6-8 after infection and administered $10^{7}$ NP-LEAPSpulsed DCs or control JH-LEAPS-pulsed DCs i.v. at 24, 48, or 72 hours after infection. Treatment with NP-LEAPS-pulsed DCs at 24 or 48 hours, but not at 72 hours, after infection, protected the infected animals from death, as demonstrated by improved percentage of survival (Figure 4A) and reduction in weight loss (Figure 4B). Virus titers were determined from the lungs of mice harvested 4 days after infection. Lower virus titers were observed in the lungs of mice that received the NP-LEAPS-pulsed DCs at 24 and 48 hours, but not 72 hours, after infection (Figure 4C). These data indicate that influenza-J-LEAPS treatment given within 48 hours following virus infection protected mice from lethal influenza virus challenge.
Influenza-J-LEAPS enhance Th1 responses in influenza infection. To determine whether LEAPS treatment could change the cytokine profile at the site of infection, we compared the cytokine profile in the lungs of infected animals after i.v. administration of NP-LEAPS, M2e-LEAPS, HA1-LEAPS, HA2-LEAPS, and combined-LEAPS-pulsed and nonpulsed DCs. In animals that exhibited a survival advantage following injection with NP-LEAPS, M2e-LEAPS, and/or combined-LEAPS-pulsed DCs (Figure 3), we found a Th1 cytokine profile with high levels of IFN- $\gamma$, IL-2, and IL-12 family cytokines. In contrast, high levels of Th2 cytokines such as IL-4 were seen in the animals that received treatment with HA1-LEAPS, HA2-LEAPS, and JH-LEAPS-pulsed DCs that were ineffective against influenza infection (Figure 5A). In addition to the cytokine profile, there was a higher PR8-specific IgG2a subclass antibody response in the sera of surviving animals at the end of the study period (day 14) that received NP-LEAPS, M2e-LEAPS, and combined-LEAPS-pulsed DCs, while control mice infected with sub-lethal doses $\left(100 \mathrm{TCID}_{50}\right)$ of PR8 maintained a bias toward a Th2 response with higher levels of PR8-specific IgG1 antibody (Figure 5B). These results confirm that the immune responses directed by influenza-J-LEAPS treatment are Th1 domi- 

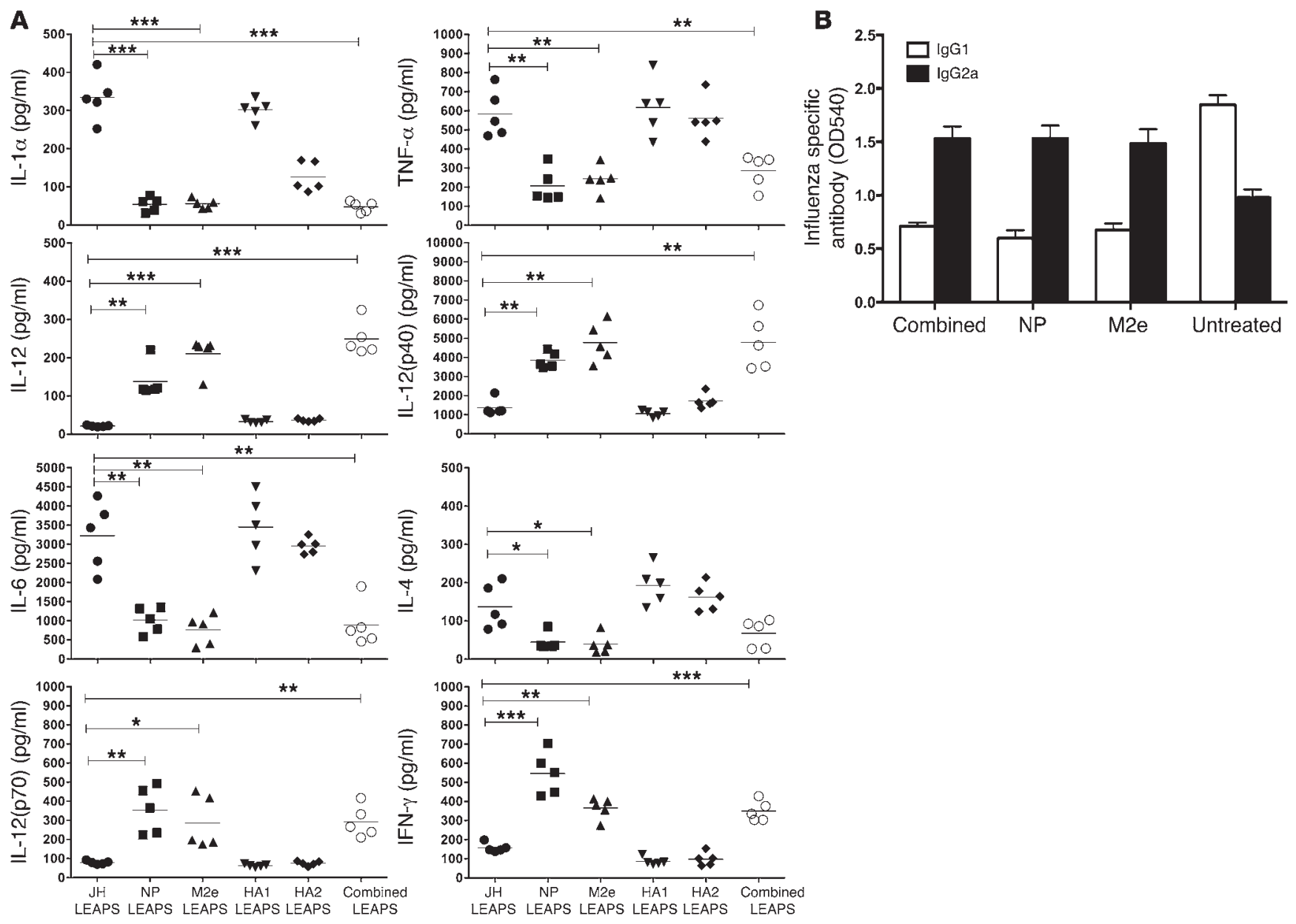

Peptides used to pulse DCs

Figure 5

LEAPS treatment results in decreased proinflammatory cytokines and increased Th1 cytokines in the lungs of PR8-infected mice (A). On day 4 after infection, lung homogenates were analyzed for cytokine protein expression. Each data point represents the result from an individual mouse; horizontal bars indicate the mean. $P$ values were determined by Student's $t$ test $\left({ }^{* * *} P<0.001 ;{ }^{* *} P<0.01\right.$; $\left.{ }^{*} P<0.05\right)$. Influenza-LEAPS elicit influenza-specific antibodies of the IgG2a subclass (B). Influenza-specific IgG1 and IgG2a subclass ELISA antibodies were determined in sera from PR8-infected mice collected on day 14 after infection. Infected mice were treated with DCs pulsed with NP-LEAPS, M2e-LEAPS, or combined-LEAPS influenza peptides. Control mice were infected i.n. with $100 \mathrm{TCID}_{50}$ of PR8, and sera were collected on day 14 after infection. The error bars represent SEM for each group.

nant. Downregulation of proinflammatory cytokines is also a hallmark of influenza-J-LEAPS treatment; a significant reduction of proinflammatory cytokines (TNF- $\alpha$, IL- $1 \alpha$, and IL-1 $\beta$ ) was observed in the lungs of animals that received the M2e-LEAPS, NP-LEAPS, and combined-LEAPS-pulsed DCs (Figure 5A).

Influenza-J-LEAPS-pulsed DCs accelerate $T$ cell responses in influenza infection. Because mature DCs specialize in antigen presentation to $\mathrm{T}$ cells, we hypothesized that administering influenza-J-LEAPSpulsed DCs would enhance T cell responses. To test this hypothesis, we administered JH-LEAPS- or NP-LEAPS-pulsed DCs to PR8infected mice $\left(10^{3} \mathrm{TCID}_{50} ; 10 \mathrm{LD}_{50}\right) 24$ hours after infection and enumerated influenza NP-specific $\left(\mathrm{NP}_{147-155}\right) \mathrm{CD}^{+} \mathrm{T}$ cells in the lungs harvested on day 4 after infection. We found a significant reduction in virus titers in the lungs of infected mice following influenza-J-LEAPS treatment (Figure 6A). We observed significant-

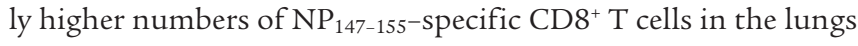

of mice that received NP-LEAPS DCs than in the lungs of mice that received JH-LEAPS (Figure 6, B and C). This phenomenon was also observed on days 5, 8, and 11 after infection (Figure 6, $\mathrm{D}$ and $\mathrm{E}$ ). These data suggest that NP-LEAPS treatment results in early recruitment of influenza-specific $\mathrm{T}$ cells into the lungs of infected mice. The number of influenza-specific $\mathrm{CD}^{+} \mathrm{T}$ cells in the lungs of animals that received NP-LEAPS-pulsed DCs remained higher than in the control group (JH-LEAPS-pulsed DCs) through 11 days after infection. These data suggest that influenza-JLEAPS-pulsed DCs enhance the magnitude of the $T$ cell responses against influenza virus at the site of infection.

Histopathology following influenza-J-LEAPS treatment. Histopathological analysis of lung tissues on day 4 after infection from JH-LEAPS- and NP-LEAPS-treated PR8-infected mice revealed multifocal moderate necrotizing bronchiolitis. This lesion was characterized by necrosis of the lining epithelial cells, often accom- 
A

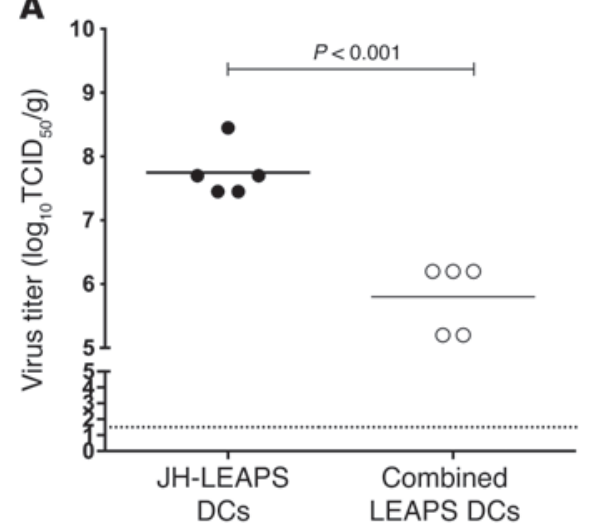

D

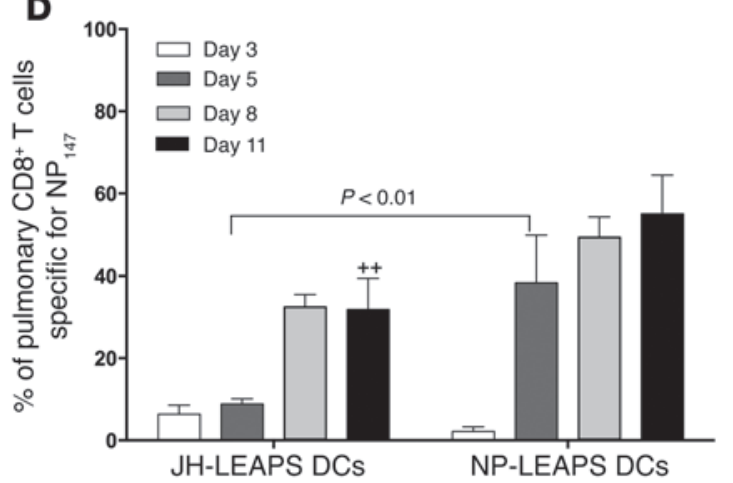

F

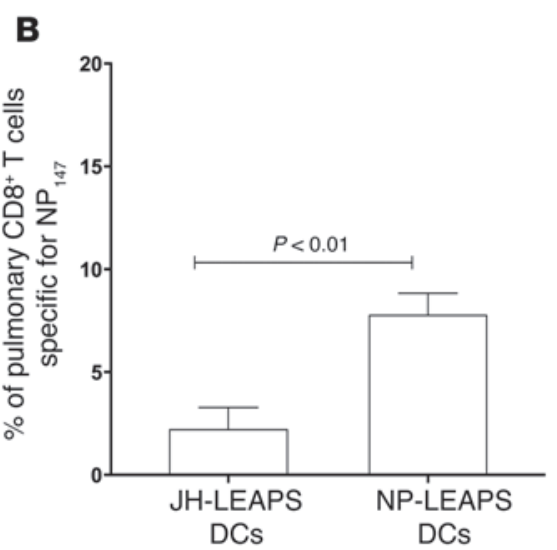

E
C

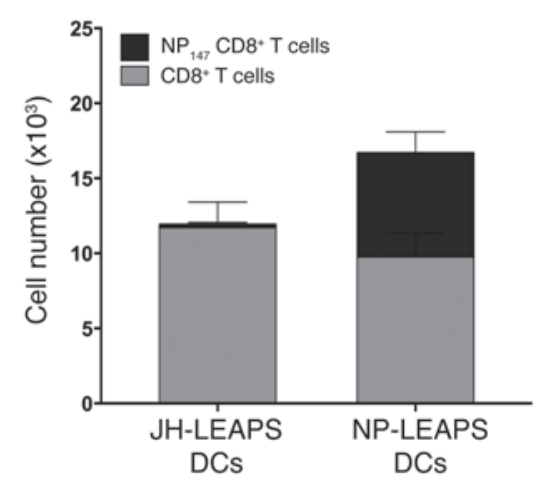

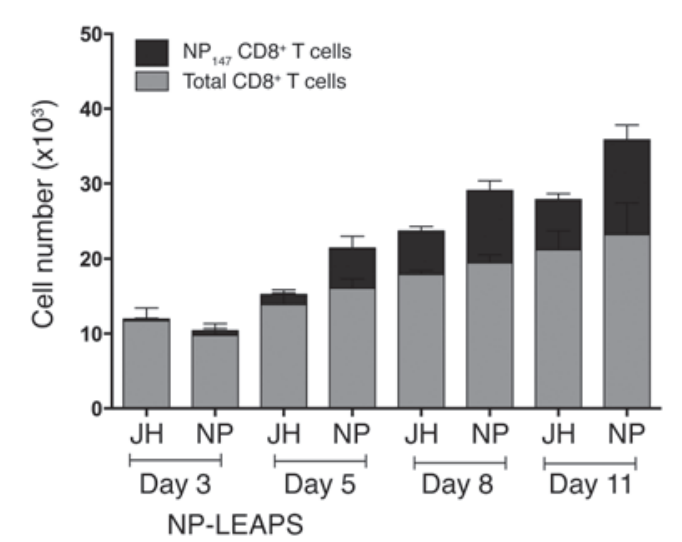

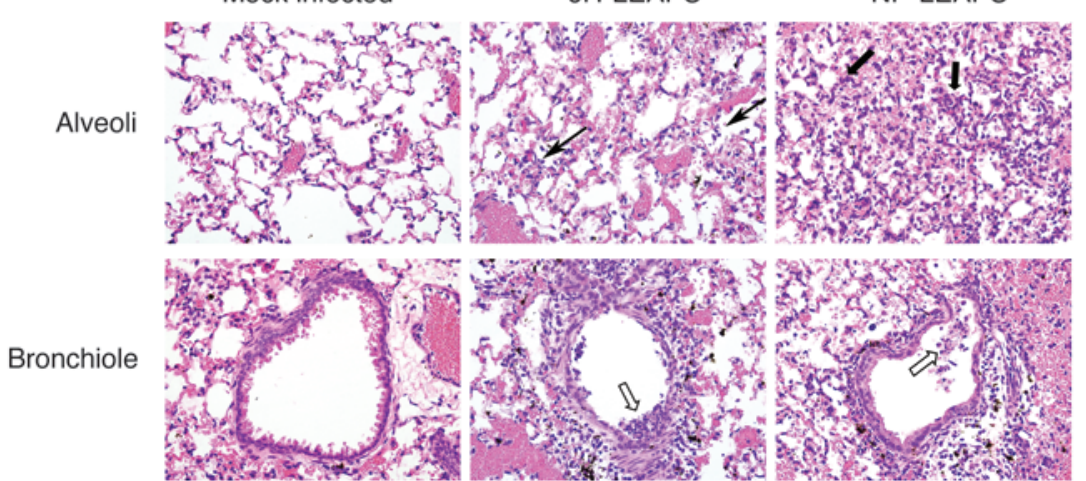

\section{Figure 6}

NP-LEAPS-pulsed DCs elicit a rapid influenza-specific CD8 ${ }^{+} \mathrm{T}$ cell response in the lungs of PR8 virus-infected mice. Mice were infected with $10 \mathrm{LD}_{50}$ of PR8 and received NP-LEAPS- or JH-LEAPS-pulsed DCs as described in Figure 1. On day 4 after infection, lungs were harvested and homogenized and virus titers were determined $(\mathbf{A})$, and isolated cells from lung tissues were stained with anti-CD3, anti-CD8, and NP 147-155 pentamer. The percentage (B) and the number $(\mathbf{C})$ of $\mathrm{CD}^{+} \mathrm{NP}_{147-155}$ pentamer ${ }^{+} \mathrm{T}$ cells were enumerated by flow cytometry. The percentage (D) and number (E) of influenza-specific CD8 ${ }^{+} T$ cells in the lungs enumerated on days 3, 5, 8, or 11 after infection are presented, except the JH-LEAPS group (indicated with ++ ), in which the animals were sacrificed on day 9 after infection because they were moribund. The error bars represent SEM for each group, $P<0.05$; Student's $t$ test. H\&E staining was performed on lung sections from $P R 8$ virus-infected mice that received JH-LEAPS DCs (middle column) or NP-LEAPS DCs (right column) 24 hours after infection. The lungs were harvested on day 4 after infection and mock-infected mice were used as control (left column) (F). Original magnification, $\times 400$.

panied by sloughing of the epithelium (Figure 6F). Affected airways frequently contained necrotic debris admixed with degenerate inflammatory cells, fibrin, and hemorrhage. Involvement of the pulmonary interstitium was minimal to mild in JH-LEAPStreated mice (Figure 6F). In contrast, NP-LEAPS-treated animals had a prominent interstitial component to the pathology that was locally extensive and severe. In the lungs of these animals, alveolar walls were thickened by mononuclear cells, primarily macrophages and lymphocytes, with type II pneumocyte hyperplasia (Figure 6F). Alveolar spaces contained moderate infiltrates of lymphocytes and macrophages and few viable neutrophils admixed with hemorrhage and occasionally necrotic cellular debris. The lungs of mock-infect- 
Rat IgG2b isotype control

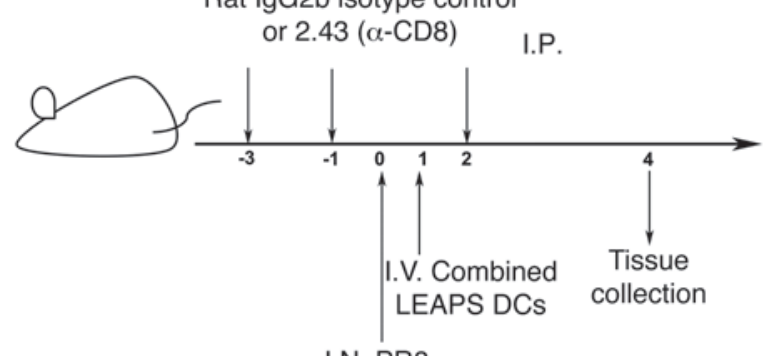

I.N. PR8

infection

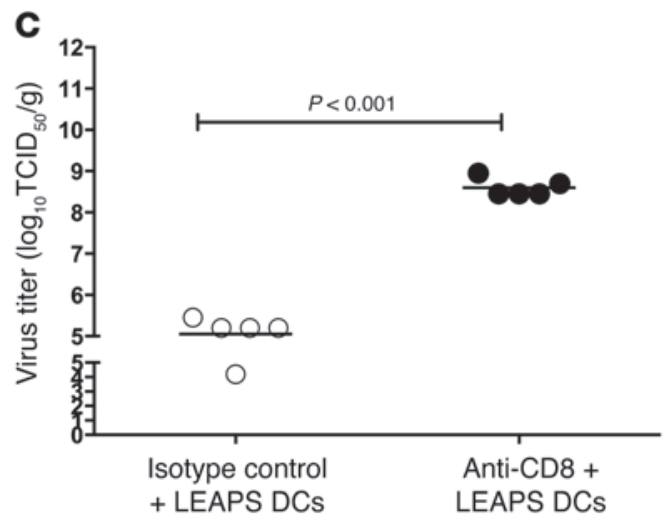

B

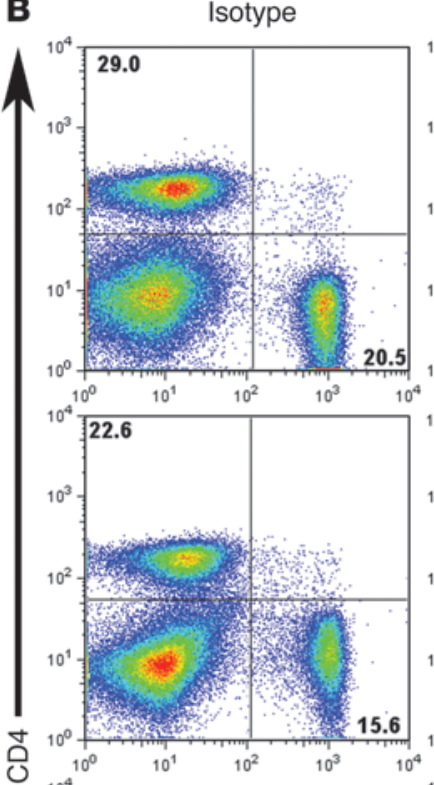

$\alpha-\operatorname{CD} 8$

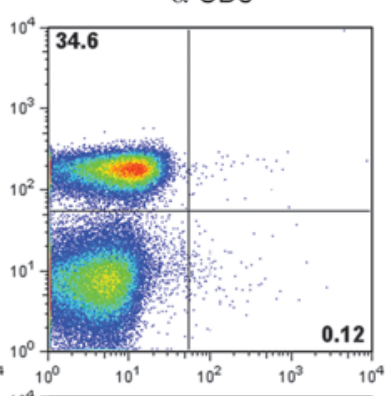

Lymph

node

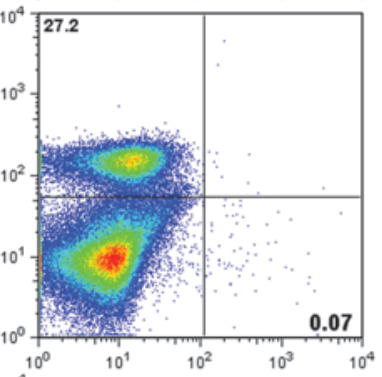

Lung
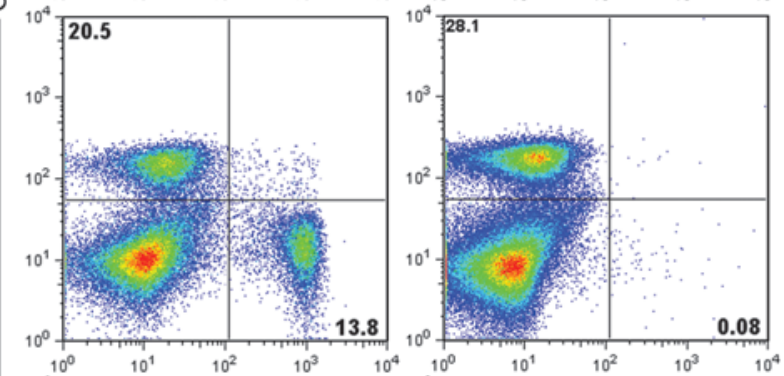

Spleen
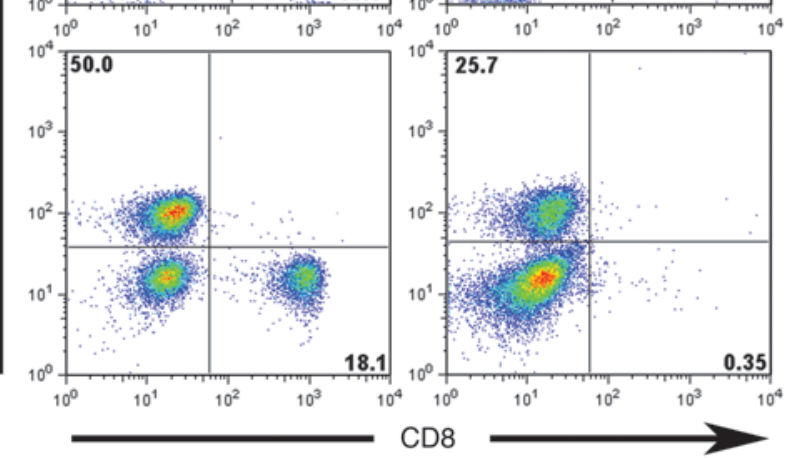

Whole

blood

\section{Figure 7}

Depletion of CD8+ $\mathrm{T}$ cells reduces the efficacy of influenza-J-LEAPS treatment. Schematic for depletion of CD8+ T cells in PR8-infected mice in combination with influenza-J-LEAPS treatment (A). FACS analysis of CD4 and CD8 expression on lymphocytes isolated from lymph nodes, lungs, spleen, and whole blood of mice treated with isotype or anti-CD8 mAbs i.p. 3 days and 1 day before and 2 days after influenza virus infection. The recorded numbers denote the percentages of $\mathrm{CD} 4^{+}$and $\mathrm{CD} 8^{+}$cells in the total lymphocyte population (B). Virus titers in the lungs were determined 4 days after infection (C). The mean titer from each group is represented as a solid bar and the dashed line represents the lower limit of detection.

ed animals were histologically normal (Figure 6F). The mononuclear cell infiltration in NP-LEAPS-treated mice was associated with reduced lung virus titers and enhanced survival, suggesting that the inflammatory response was associated with virus clearance.

Depletion of $C D 8^{+} T$ cells reduces the efficiency of influenza-J-LEAPS treatment. To evaluate the role of $\mathrm{CD}^{+} \mathrm{T}$ cells in influenza-J-LEAPS treatment, we depleted $\mathrm{CD}^{+} \mathrm{T}$ cells before and after influenza-JLEAPS treatment by i.p. injection of a $\mathrm{mAb}$ against murine $\mathrm{CD} 8^{+} \mathrm{T}$ cells; the control group received an isotype control mAb (Figure 7A). The completeness of $\mathrm{CD}^{+} \mathrm{T}$ cell depletion was determined by flow cytometry; FACS analysis revealed nearly complete depletion of
$\mathrm{CD}^{+} \mathrm{T}$ cells from the lymph nodes, lungs, spleen, and whole blood (Figure 7B). On day 4 after infection, reduced replication of PR8 virus was observed in the lungs of mice that received the isotype control $\mathrm{mAb}$ in combination with influenza-J-LEAPS treatment, but not in the lungs of mice depleted of $\mathrm{CD}^{+} \mathrm{T}$ cells (Figure 7C), suggesting that $\mathrm{CD}^{+} \mathrm{T}$ cells play a critical role in the clearance of virus following influenza-J-LEAPS treatment.

NP-LEAPS DC treatment is effective in treating oseltamivir-resistant pH1N1 virus-infected mice. Given the limited therapeutic options for infection caused by influenza viruses resistant to available antiviral drugs, we chose to evaluate the use of NP-LEAPS-stimu- 
A
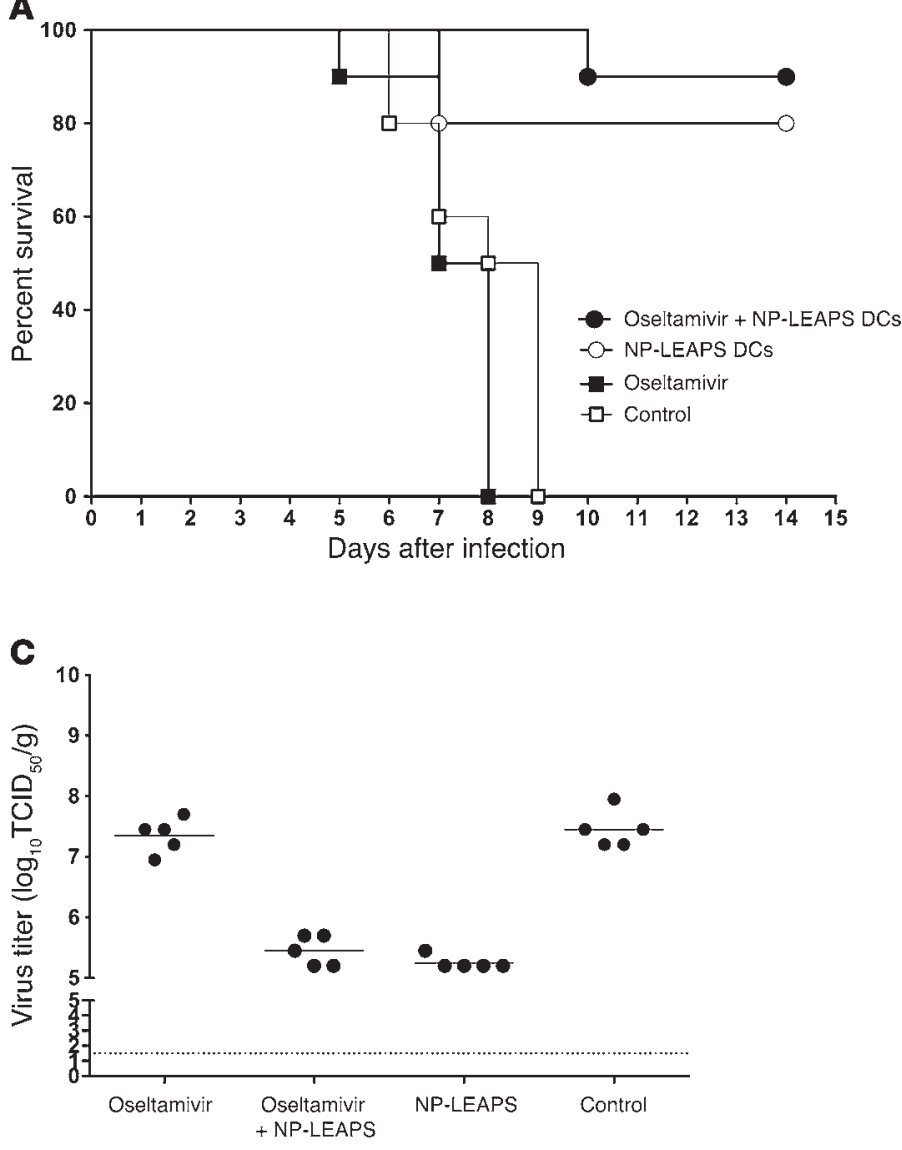

B

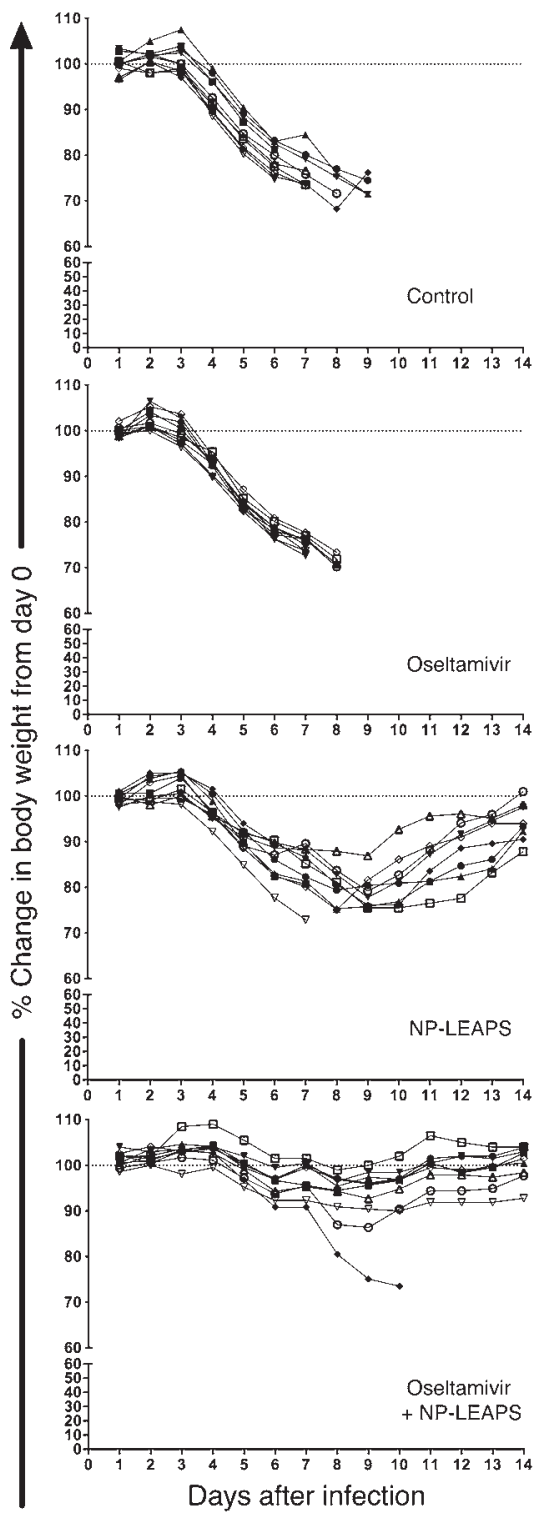

\section{Figure 8}

NP-LEAPS DC treatment is effective in mice infected with an oseltamivir- resistant virus. Mice infected with $10^{6} \mathrm{TCID}{ }_{50}$ of $\mathrm{A} / \mathrm{Bethesda/NIH107-}$ D31/2009 (oseltamivir-resistant pH1N1) were treated with oseltamivir phosphate $(20 \mathrm{mg} / \mathrm{kg} / \mathrm{d}$ ) twice daily for 5 days starting 4 hours before infection. Oseltamivir was given alone or combined with NP-LEAPS pulsed DCs, which were given 24 hours after infection. The control group received sterile water by oral gavage twice daily for 5 days and $200 \mu$ l sterile PBS i.v. 24 hours after infection. Percentage of survival (A), percentage of changes in body weight (B), and virus titer in lungs (C) harvested 6 days after infection are presented.

lated DCs to treat oseltamivir-resistant $\mathrm{pH} 1 \mathrm{~N} 1$-infected mice. We infected mice with $10^{6} \mathrm{TCID}_{50}$ of a clinical isolate of $\mathrm{pH} 1 \mathrm{~N} 1$ that was oseltamivir resistant (24). Mice were orally gavaged with $20 \mathrm{mg} / \mathrm{kg} / \mathrm{d}$ of the prodrug oseltamivir phosphate (oseltamivir) for 5 days starting 4 hours before infection; this dose is reported to produce a plasma concentration comparable to that of the recommended human oral dose (75 mg twice daily) (25). In addition to oseltamivir treatment, a group of animals $(n=15)$ was given NP-LEAPS-stimulated DCs i.v. 24 hours after infection. An infected, untreated control group received distilled water by oral gavage twice daily for 5 days and $200 \mu$ l PBS i.v. 24 hours after infection. Oseltamivir treatment did not protect mice from lethal infection with the oseltamivir-resistant $\mathrm{pH} 1 \mathrm{~N} 1$ virus, whereas protection was observed in the groups that received NP-LEAPS DCs alone and combination treatment (oseltamivir + NP-LEAPS DCs) (Figure 7A). Virus titers in the lungs of treated and untreated mice were determined on day 6 after infection. Virus titers in the lungs of mice were similar in oseltamivir-treated and untreated (control) mice, indicating that oseltamivir failed to reduce replication of the virus in the lungs of infected animals. In contrast, the virus titers in the lungs of animals that received NP-LEAPS DCs alone or NP-LEAPS DCs combined with oseltamivir were significantly lower than in the untreated control group (Figure 7B). Infected, untreated control, and oseltamivir-treated mice lost weight rap- 
idly and continued to lose weight and died between days 7 and 10 after infection. The mice that received NP-LEAPS DCs alone began regaining weight by day 10 after infection. Interestingly, the mice that received combined treatment (NP-LEAPS DCs and oseltamivir) maintained their weight through the course of infection better than other the groups (Figure $7 \mathrm{C}$ ). These results demonstrate that NP-LEAPS-stimulated DCs were effective in treating infection with an antiviral drug-resistant influenza virus.

\section{Discussion}

We demonstrated the efficacy of what we believe to be a novel immune-based therapy for influenza that uses LEAPS technology to deliver influenza antigens via DCs. We found that influenza-JLEAPS protect mice from lethal infection with 10 and $1000 \mathrm{LD}_{50}$ of PR8 (Figure 1) and pH1N1 influenza viruses (Supplemental Figure 3). Our study results are in agreement with and substantially extend previous reports that showed that LEAPS-conjugated viral peptides can protect animals from lethal HSV infection (15-17). However, in this study, LEAPS was used for therapy rather than prevention and our results demonstrate that peptide-conjugated LEAPS stimulates the maturation of DCs (Supplemental Figure 2 ), which are important for antigen presentation and are critical for $T$ cell activation. The usefulness of this approach for the treatment of influenza in humans warrants investigation. Although ferrets are widely used as a model for the study of influenza, the lack of immunological reagents is a major obstacle to studying the efficacy of influenza-J-LEAPS DCs in ferrets.

We found that influenza-J-LEAPS-stimulated DCs were recruited to the lungs of infected mice but not to the lungs of uninfected mice (Figure 2). The ligand and the mechanism by which J-LEAPS-conjugated peptides stimulate DC maturation requires further investigation. Possible mechanisms have been studied in a model of HSV (10). Our current hypothesis is that LEAPS initiates DC activation by binding to a receptor on a precursor cell; this promotes maturation to semi-mature DCs that have the potential to mature further. LEAPS DCs produce IL-12p70 and present the loaded peptide to $\mathrm{T}$ cells to promote IFN- $\gamma$ production and Th1 responses. Interestingly, one feature that makes the LEAPS DCs unique is their ability to make IL-12p70 without TNF- $\alpha$, which promotes inflammation. IL-12p70 in the absence of TNF- $\alpha$ appears to activate Th1 responses without excessive and pathogenic inflammatory responses (26).

In the current study, early recruitment and a greater magnitude of influenza virus-specific $\mathrm{T}$ cells were detected in the lungs of NP-LEAPS DC-treated animals (Figure 6, B-E), suggesting that LEAPS-stimulated DCs exert their effect by directly migrating to the site of infection where they can bypass antigen processing and efficiently prime $\mathrm{T}$ cells. We found that depletion of $\mathrm{CD}^{+} \mathrm{T}$ cells in vivo reduced the efficacy of influenza-J-LEAPS treatment (Figure 7), suggesting a critical role for influenza-specific $\mathrm{CD}^{+}$ $\mathrm{T}$ cells in this scenario. It is likely that coupling peptides to the $\beta_{2}$-microglobulin subunit of MHC class I (J-LEAPS ligand) increases the stability of the MHC/peptide complex (11-13) thereby eliciting strong $\mathrm{T}$ cell responses in treated animals. Histologic evidence of lymphocyte and macrophage infiltration (Figure 6F) at the site of infection suggests that a cell-mediated immune response is the hallmark of LEAPS-stimulated DC treatment.

In general, the $\mathrm{T}$ cell response to influenza virus is initially activated in the lymph nodes, and influenza-specific $T$ cells are observed at the site of infection (i.e., the lung airways and lung parenchyma) about 5-7 days after infection (27). However, we found that influenza-J-LEAPS treatment results in early recruitment of influenza-specific T cells to the site of infection. The kinetics with LEAPS-pulsed DCs may be different for 2 reasons. First, the response to small peptides, which could be considered as preprocessed materials, may be different from the response to intact whole infectious virus. Second, we administered ex vivo matured J-LEAPS DCs, which home to the lungs rapidly and may recruit $\mathrm{T}$ cells much earlier than would be expected in a typical influenza virus infection.

Although the various influenza peptides we tested were conjugated to $\beta_{2}$-microglobulin, only NP and M2e peptides were suitable and appeared to be critical for the efficacy of LEAPS-DC treatment (Figure 3) against lethal infection with 2 different strains of influenza (PR8 and $\mathrm{pH} 1 \mathrm{N1}$ ). Our data establish the potential of these 2 peptides in the context of LEAPS as an attractive target for broad-spectrum immune-based therapy. We found that HAconjugated LEAPS did not induce maturation of DCs as well as the other peptides (Supplemental Figure 3). We believe this might explain the reduced ability of the HA-conjugated LEAPS DCs to stimulate $\mathrm{T}$ cell responses. This result suggests that the ability of peptide-conjugated LEAPS to induce DC maturation is critical for the success of LEAPS treatment.

In addition, we demonstrated the effectiveness of NP-LEAPS DC treatment of oseltamivir-resistant $\mathrm{pH} 1 \mathrm{~N} 1$ virus infection (Figure 8). Although oseltamivir treatment alone did not protect the infected mice from lethal challenge, animals that received a combination of oseltamivir and NP-LEAPS DC treatment maintained their weight better than the other groups; the reason for this effect is not known.

The severity of influenza is mediated by both viral and host factors. Our study provides evidence that LEAPS treatment limited the excessive cytokine and chemokine production often associated with severe influenza virus infection (also known as "cytokine storm”) (20). The delivery of NP-LEAPS, M2e-LEAPS, and combined-LEAPS-pulsed DCs reduced the levels of proinflammatory cytokines associated with influenza virus-induced pathology (Figure 4). This effect is similar to treatment with $\mathrm{S}_{1} \mathrm{P}_{1}$ agonists that suppress cytokine and chemokine production in the lungs of influenza-infected mice (28-32). In our study, in addition to the dampened inflammatory cytokine response, influenza-J-LEAPS DCs steered the immune response toward Th1, with increased Th1 signature cytokines, e.g., IL-12, IL-2, and IFN- $\gamma(33,34)$, and a shift of influenza-specific antibodies toward an IgG2a subclass response, whereas the untreated-infected mice had a dominant IgG1 subclass response (Figure 6B). These data are in agreement with the results obtained from LEAPS treatment in HSV and malaria (35).

Although LEAPS treatment showed a profound effect on influenza virus-infected mice, this treatment has at least one marked limitation. LEAPS-pulsed DCs had to be administered 24 to 48 hours after infection (Figure 4 ) to be able to affect morbidity (as measured by weight loss) and survival of influenza-infected (PR8 and pH1N1) mice. This narrow time window would present an important challenge, since the therapeutic window in human influenza virus infection is usually narrow. Despite concerns about the applicability of this approach in humans based on time constraints, we remain hopeful that the method will be viable for several reasons. First, the present protocol starts with murine BM and not immature DCs or monocytes, and the 8-day expansion/maturation period with GM-CSF is needed to get enough 
cells for subsequent J-LEAPS activation. Unlike with mice, it is possible to obtain monocytes from the peripheral circulation in humans. Therefore, the 8-day treatment that was necessary for mouse BM cells would probably not be necessary with human cells. Second, it may be possible to reduce the time to treat mouse BM cells. In a mouse model of HSV infection, mouse BM cells treated with a J-LEAPS HSV vaccine for only 24 hours provided protection from lethal infection (21). Third, we have previously demonstrated that human peripheral blood monocytes can produce IL-12p70, MCP1, MCP2, and Rantes within 24 hours without the need for GM-CSF or GM-CSF + IL-4 (36) and would most likely use this approach in a clinical setting. Additionally, it is possible that treatment with LEAPS-loaded DCs can be combined with antiviral drugs, since LEAPS-stimulated DCs have a different purported mechanism of action. We have demonstrated the benefits of treatment with LEAPS-loaded DCs on influenza virus infection in a small animal model; the efficacy and usefulness of this approach for the treatment of influenza virus in humans warrants investigation.

In summary, we have demonstrated that modulation of immune responses by LEAPS technology results in significantly reduced mortality and morbidity during influenza infection in mouse models of influenza (PR8 and pH1N1).

\section{Methods}

Mice. Male and female BALB/c mice (8 to 10 weeks old) were obtained from Taconic (Taconic Farms, Inc.). All animal experiments were performed at the NIH, in compliance with the guidelines of the NIAID, NIH Institutional Animal Care and Use Committee.

Virus. PR8 virus was amplified in the allantoic cavity of 10-day-old specific pathogen-free embryonated chicken eggs, aliquoted, and stored at $-80^{\circ} \mathrm{C}$. The titer of the virus stock was determined in MDCK cells and is expressed as a $50 \%$ tissue culture infectious dose $\left(\mathrm{TCID}_{50}\right)$.

The WT pandemic H1N1 virus A/California/7/2009 (CA09wt) (pH1N1) was kindly provided by the Influenza Division, Centers for Disease Control and Prevention (CDC). The CA09wt virus was propagated in the allantoic cavity of 9- to 11-day-old embryonated specific pathogen-free hen's eggs. The titer of the virus was determined in Madin-Darby canine kidney (MDCK) cells. Allantoic fluid from passage 4 was used in this study. There are 2 amino acid differences (N125D and Q223R) between the HA proteins of the CA09wt virus used in this study and those available in GenBank (FJ969540.1).

Oseltamivir resistant virus A/Bethesda/NIH107-D31/2009 was provided by Matthew J. Memoli (NIAID, NIH) (24). The virus was propagated and the titer of virus was determined in MDCK cells.

LEAPS design and synthesis. The influenza-J-LEAPS consist of an immune cell-binding ligand, DLLKNGERIEKVE, from amino acids 38-50 of $\beta_{2}$-microglobulin conjugated to a peptide from the NP, M2, or HA protein of influenza A virus (Supplemental Table 1). The influenza-J-LEAPS were synthesized and purified by CEL-SCI.

Generation of murine BMDCs. BMDCs were generated as previously described $(37,38)$ with some modifications. Briefly, BM progenitor cells collected from the tibias and femurs of male BALB/c mice were passed through a nylon mesh to remove small pieces of bone and debris, resuspended in complete medium (RPMI 1640 [Invitrogen] containing 10\% FCS [Hyclone], $50 \mu \mathrm{M}$ 2-ME [Sigma-Aldrich], $2 \mathrm{mM}$ glutamine [Invitrogen], and $0.1 \mathrm{mM}$ nonessential amino acids [Cellgro Mediatech Inc.]) supplemented with $20 \mathrm{ng} / \mathrm{ml}$ recombinant murine GM-CSF (PepProTech Inc.) and cultured in tissue culture plates (BD). Nonadherent cells were removed on day 2 , and adherent cells were washed and re-fed with fresh complete medium containing $20 \mathrm{ng} / \mathrm{ml}$ murine GM-CSF every 2 days. On day 8 of culture, most of the nonadherent cells had acquired typical dendritic morphology; DC phenotypes were confirmed by flow cytometry, and these cells were used as the source of DCs in subsequent experiments.

DC maturation. Influenza-J-LEAPS were added to DCs at a $14.5 \mu \mathrm{M}$ concentration. DC maturation markers were confirmed by flow cytometry.

Cell labeling with CFSE. DCs were resuspended in PBS containing $0.1 \%$ bovine albumin (Sigma-Aldrich). $10^{7}$ cells $/ \mathrm{ml}$ were incubated with $5 \mu \mathrm{M}$ $\mathrm{CFSE}$ (Invitrogen) at $37^{\circ} \mathrm{C}$ for 10 minutes. Labeled cells were washed twice with PBS/0.1\% BSA and resuspended in sterile PBS.

Influenza virus infection and treatment of mice. Mice were anesthetized with $4 \%$ isoflurane, followed by i.n. administration of $10^{5}$ or $10^{3} \mathrm{TCID}_{50}$ of PR8 in $50 \mu \mathrm{l}$ L-15 medium. $10^{7}$ unstimulated or LEAPS-stimulated DCs in $200 \mu \mathrm{l}$ sterile PBS were given i.v. 24 hours following infection unless otherwise indicated.

Flow cytometry. A FACSCalibur instrument (BD Biosciences) was used to monitor cell-surface staining. For phenotyping of DCs, cells were stained with CD3-PerCP, CD19-PE, class II-PE, CD86-APC, CD11c-Alexa Fluor 488, CD80-PE, and F4/80-FITC (BD Bioscience).

Pentamer staining. Pentamers (H-K2d) were loaded with peptides corresponding to residues 147 to 155 of nucleocapsid protein NP ( $\left.\mathrm{NP}_{147-155}\right)$; TYQRTRALV-conjugated PE was purchased from ProImmune (ProImmune Inc.). Cell staining with pentamers was performed according to manufacturer's instructions.

Viral titration assay. Lungs were harvested, weighed, and homogenized in L-15 medium (Hyclone) to prepare a 10\% (w/v) tissue homogenate. The homogenates were clarified by low-speed centrifugation, and the viral titers determined on MDCK monolayers were expressed as $\log _{10} \mathrm{TCID}_{50} / \mathrm{g}$ of tissue (39).

Cytokine analysis. The concentration of various cytokines and chemokines in clarified lung homogenates was determined by a Bio-Plex Protein Array system (Bio-Rad). The assay was performed according to manufacturer's instructions. The results were analyzed using Bio-Plex manager software.

ELISA. $\beta$-Propiolactone-inactivated (BPL-inactivated) whole virus was used for ELISA as previously described $(40,41)$.

Pathology. Animals were euthanized on day 4 after infection by $\mathrm{CO}_{2}$ inhalation and necropsied according to a standard protocol. The lungs were inflated with $10 \%$ neutral buffered formalin, embedded in paraffin, sectioned at $4 \mu \mathrm{m}$, and stained with H\&E for examination by light microscopy. As a control for histopathology, 2 mice were sham inoculated with PBS and sacrificed at the same time point. Slides were examined by a pathologist without knowledge of the treatment group.

Combination of oseltamivir and LEAPS treatment in vivo. Female 6- to 8-weekold BALB/c mice (weight 18-20 g) were used in the experiment. Mice were anesthetized by inhalation of isoflurane and inoculated i.n. with $50 \mu \mathrm{l}$ of $10^{6}$ TCID $_{50} \mathrm{~A} /$ Bethesda/NIH107-D31/2009 (H1N1), an oseltamivir-resistant virus. Treatment with oseltamivir phosphate $(20 \mathrm{mg} / \mathrm{kg} / \mathrm{d}$ by twicedaily oral gavage; Toronto Research Chemicals Inc.) was initiated 4 hours before infection and continued for 5 days. A group of mice $(n=15)$ was given $10^{7}$ cells of NP-LEAPS-stimulated DCs 24 hours after infection i.v. in combination with oseltamivir phosphate treatment by oral gavage. In this experiment, the lung tissues were harvested for virus titer on day 6 after infection after oseltamivir treatment was complete. Influenza disease severity was measured by morbidity and mortality; in addition, virus titers in the lungs were quantified.

In vivo depletion of $\mathrm{CD} 8^{+} \mathrm{T}$ cells. In vivo $\mathrm{CD} 8^{+} \mathrm{T}$ cell depletion was conducted as previously described (42). Briefly, depletion of CD8 ${ }^{+} \mathrm{T}$ cells was performed before and after infection by i.p. injection of mAbs against $\mathrm{CD} 8^{+} \mathrm{T}$ cells. The completeness of the depletion was determined in lymph nodes, lungs, spleen, and whole blood on day 4 after infection by flow cytometry. Clone 53.6.7 (anti-CD8) was used for cell staining, which is different from the anti-CD8 (2.43) clone used for T cell depletion. 
Statistics. The significance of differences between different groups was assessed with the unpaired Student's $t$ test and log-rank (Mantel-cox) test using Prism 5 (GraphPad Software, Inc.). $P<0.05$ was considered significantly different.

Study approval. All animal studies were approved by the NIAID, NIH Institutional Animal Care and Use Committee.

\section{Acknowledgments}

This research was supported in part by the Intramural Research Program of the NIAID, NIH. All the peptides tested in this project were supplied by CEL-SCI Corp. We would like to thank Mat- thew Memoli and Jeffery Taubenberger for providing A/Bethesda/ NIH107-D31/2009 (H1N1), the oseltamivir-resistant virus.

Received for publication October 26, 2012, and accepted in revised form April 11, 2013.

Address correspondence to: Kanta Subbarao, Emerging Respiratory Viruses Section, Laboratory of Infectious Diseases, NIAID, NIH, Bldg. 33, Room 3E13C.1, 33 North Drive, MSC 3203, Bethesda, Maryland 20892-3203, USA. Phone: 301.451.3839; Fax: 301.480.4749; E-mail: KSUBBARAO@niaid.nih.gov.
1. Govorkova EA, McCullers JA. Therapeutics against influenza. Curr Top Microbiol Immunol. 2013; 370:273-300.

2. van der Vries E, Schutten M, Boucher CA. The potential for multidrug-resistant influenza. Curr Opin Infect Dis. 2011;24(6):599-604.

3. Baranovich T, Webster RG, Govorkova EA. Fitness of neuraminidase inhibitor-resistant influenza A viruses. Curr Opin Virol. 2011;1(6):574-581.

4. Writing Committee of the Second World Health Organization Consultation on Clinical Aspects of Human Infection with Avian Influenza A (H5N1) Virus, et al. Update on avian influenza A (H5N1) virus infection in humans. N Engl J Med. 2008; 358(3):261-273.

5. Le QM, et al. Avian flu: isolation of drug-resistant H5N1 virus. Nature. 2005;437(7062):1108.

6. Storms AD, et al. Oseltamivir-resistant pandemic (H1N1) 2009 virus infections, United States, 20102011. Emerg Infect Dis. 2012;18(2):308-311.

7. Yen HL, Ilyushina NA, Salomon R, Hoffmann E, Webster RG, Govorkova EA. Neuraminidase inhibitor-resistant recombinant A/Vietnam/1203/04 (H5N1) influenza viruses retain their replication efficiency and pathogenicity in vitro and in vivo. J Virol. 2007;81(22):12418-12426.

8. Hayden F. Developing new antiviral agents for influenza treatment: what does the future hold? Clin Infect Dis. 2009;48(suppl 1):S3-S13.

9. Parham P, Androlewicz MJ, Holmes NJ, Rothenberg BE. Arginine 45 is a major part of the antigenic determinant of human beta 2-microglobulin recognized by mouse monoclonal antibody BBM.1. J Biol Chem. 1983;258(10):6179-6186.

10. Rosenthal KS, Taylor P, Zimmerman DH. J-LEAPS peptide and LEAPS dendritic cell vaccines. Microb Biotechnol. 2012;5(2):203-213.

11. Uger RA, Barber BH. Creating CTL targets with epitope-linked beta 2 -microglobulin constructs. J Immunol. 1998;160(4):1598-1605.

12. Uger RA, Chan SM, Barber BH. Covalent linkage to beta2-microglobulin enhances the MHC stability and antigenicity of suboptimal CTL epitopes. J Immunol. 1999;162(10):6024-6028.

13. White J, Crawford F, Fremont D, Marrack P, Kappler J. Soluble class I MHC with beta2-microglobulin covalently linked peptides: specific binding to a T cell hybridoma. J Immunol. 1999;162(5):2671-2676.

14. Zimmerman DH, Rosenthal KS. The L.E.A.P.S. approach to vaccine development. Front Biosci. 2005; 10:790-798.

15. Rosenthal KS, Mao H, Horne WI, Wright C, Zimmerman D. Immunization with a LEAPS heteroconjugate containing a CTL epitope and a peptide from beta-2-microglobulin elicits a protective and DTH response to herpes simplex virus type 1.
Vaccine. 1999;17(6):535-542.

16. Goel N, Rong Q, Zimmerman D, Rosenthal KS. A L.E.A.P.S. heteroconjugate vaccine containing a T cell epitope from HSV-1 glycoprotein D elicits Th 1 responses and protection. Vaccine. 2003 ; 21(27-30):4410-4420.

17. Goel N, Zimmerman DH, Rosenthal KS. Ligand epitope antigen presentation system vaccines against herpes simplex virus. Front Biosci. 2005; 10:966-974.

18. Tscherne DM, Garcia-Sastre A. Virulence determinants of pandemic influenza viruses. $J$ Clin Invest. 2011;121(1):6-13.

19. Kobasa D, et al. Aberrant innate immune response in lethal infection of macaques with the 1918 influenza virus. Nature. 2007;445(7125):319-323.

20. de Jong MD, et al. Fatal outcome of human influenza A (H5N1) is associated with high viral load and hypercytokinemia. Nat Med. 2006;12(10):1203-1207.

21. Taylor PR, et al. J-LEAPS vaccines initiate murine Th1 responses by activating dendritic cells. Vaccine. 2010;28(34):5533-5542.

22. Cihakova D, et al. L.E.A.P.S. heteroconjugate is able to prevent and treat experimental autoimmune myocarditis by altering trafficking of autoaggressive cells to the heart. Int Immunopharmacol. 2008; 8(5):624-633.

23. Zimmerman DH, et al. CEL-2000: A therapeutic vaccine for rheumatoid arthritis arrests disease development and alters serum cytokine/chemokine patterns in the bovine collagen type II induced arthritis in the DBA mouse model. Int Immunopharmacol. 2010;10(4):412-421.

24. Memoli MJ, et al. Multidrug-resistant 2009 pandemic influenza $\mathrm{A}(\mathrm{H} 1 \mathrm{~N} 1)$ viruses maintain fitness and transmissibility in ferrets. J Infect Dis. 2010; 203(3):348-357.

25. Govorkova EA, Ilyushina NA, McClaren JL, Naipospos TS, Douangngeun B, Webster RG. Susceptibility of highly pathogenic H5N1 influenza viruses to the neuraminidase inhibitor oseltamivir differs in vitro and in a mouse model. Antimicrob Agents Chemother. 2009;53(7):3088-3096.

26. Zimmerman DH, Steiner H 3rd, Carmabula R, Talor E, Rosenthal KS. LEAPS therapeutic vaccines as antigen specific suppressors of inflammation in infectious and autoimmune diseases. J Vaccines Vaccin. 2012;3(5):149.

27. Kohlmeier JE, Woodland DL. Immunity to respiratory viruses. Annu Rev Immunol. 2009;27:61-82.

28. Marsolais D, et al. Local not systemic modulation of dendritic cell S1P receptors in lung blunts virusspecific immune responses to influenza. Mol Pharmacol. 2008;74(3):896-903.

29. Marsolais D, et al. A critical role for the sphingosine analog AAL- $\mathrm{R}$ in dampening the cytokine response during influenza virus infection. Proc Natl Acad Sci U S A. 2009;106(5):1560-1565.

30. Walsh KB, et al. Suppression of cytokine storm with a sphingosine analog provides protection against pathogenic influenza virus. Proc Natl Acad SciU S A. 2011;108(29):12018-12023.

31. Walsh KB, Teijaro JR, Rosen H, Oldstone MB. Quelling the storm: utilization of sphingosine-1phosphate receptor signaling to ameliorate influenza virus-induced cytokine storm. Immunol Res. 2011; 51(1):15-25.

32. Teijaro JR, et al. Endothelial cells are central orchestrators of cytokine amplification during influenza virus infection. Cell. 2011;146(6):980-991.

33. Pulendran B. Modulating TH1/TH2 responses with microbes, dendritic cells, and pathogen recognition receptors. Immunol Res. 2004;29(1-3):187-196.

34. Monteiro JM, Harvey C, Trinchieri G. Role of interleukin-12 in primary influenza virus infection. J Virol. 1998;72(6):4825-4831.

35. Charoenvit Y, Goel N, Whelan M, Rosenthal KS, Zimmerman DH. CEL-1000 - a peptide with adjuvant activity for Th1 immune responses. Vaccine. 2004;22(19):2368-2373.

36. Taylor PR, Paustian CC, Koski GK, Zimmerman $\mathrm{DH}$, Rosenthal KS. Maturation of dendritic cell precursors into IL12-producing DCs by J-LEAPS immunogens. Cell Immunol. 2010;262(1):1-5.

37. Inaba $\mathrm{K}$, et al. Generation of large numbers of dendritic cells from mouse bone marrow cultures supplemented with granulocyte/macrophage colony-stimulating factor. J Exp Med. 1992; 176(6):1693-1702.

38. Lutz MB, et al. An advanced culture method for generating large quantities of highly pure dendritic cells from mouse bone marrow. I Immunol Methods. 1999;223(1):77-92.

39. Joseph T, McAuliffe J, Lu B, Jin H, Kemble G, Subbarao K. Evaluation of replication and pathogenicity of avian influenza a $\mathrm{H} 7$ subtype viruses in a mouse model. J Virol. 2007;81(19):10558-10566.

40. Lau YF, Tang LH, McCall AW, Ooi EE, Subbarao $\mathrm{K}$. An adjuvant for the induction of potent, protective humoral responses to an $\mathrm{H} 5 \mathrm{~N} 1$ influenza virus vaccine with antigen-sparing effect in mice. $J$ Virol. 2010;84(17):8639-8649.

41. Lau YF, Santos C, Torres-Velez FJ, Subbarao K. The magnitude of local immunity in the lungs of mice induced by live attenuated influenza vaccines is determined by local viral replication and induction of cytokines. J Virol. 2011;85(1):76-85.

42. Chen J, et al. Cellular immune responses to severe acute respiratory syndrome coronavirus (SARS$\mathrm{CoV}$ ) infection in senescent BALB/c mice: CD4+ T cells are important in control of SARS-CoV infection. J Virol. 2010;84(3):1289-1301. 